\title{
ÉTICA PROFESIONAL
}

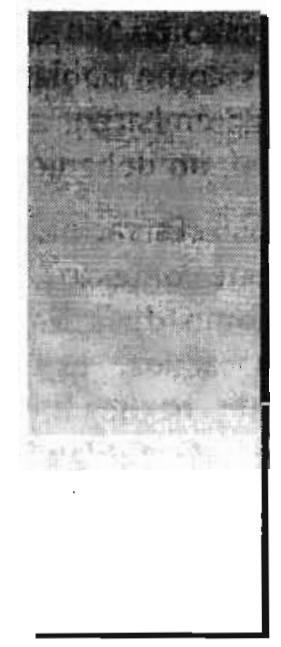

MIGUEL ÁNGEL POLO SANTILLÁN*

E-mail:polotzu@yahoo.com

\section{INTRODUCCIÓN}

Antes de iniciar el tema, quisiera plantearme la siguiente pregunta: ¿en qué contexto hablamos de ética profesional?, ¿cuál es la importancia de este tema en nuestro contexto nacional? Sabemos que la corrupción social se ha agudizado desde los años 90 , en la cual han participado profesionales de distintas carreras como de diversas universidades y estratos sociales. El espacio político terminó corrompiendo la actividad profesional. Ante tal evento, la actividad profesional debe recobrar su sentido a partir de la afirmación de su propio espacio, que es el espacio público. Desde ahí puede ayudar a recobrar el sentido de la moral social tan venida a menos. No es, pues, este tema uno entre otros, sino uno que afecta al entramado social.

Entender el papel de la ética en las profesiones requiere entender las profesiones, su devenir y naturaleza. Desde ahí podremos comprender que hablar de ética profesional no es simplemente un asunto adicional de buena voluntad, sino que es aquello que le da sentido a la práctica profesional. Por eso nuestro artículo quiere esclarecer el concepto y al hacerlo quedará clara la función de la ética profesional. Así, dividiré el artículo en las siguientes partes: i) presentar el significado de la ética en la vida humana; ii) el bosquejo histórico de las profesiones; iii) el sentido de la profesión; iv) la profesión como una actividad con fines internos; v) las excelencias necesarias para lograr los fines; vi) entender la profesión como una actividad comunitaria; vii) tomar conciencia de que las profesiones forman parte de la sociedad civil; viii) finalmente, presentaremos la conclusión de lo trabajado. El desarrollo no quiere ser sólo expositivo, sino también veremos las posibles corrupciones o problemas que puede afrontar la actividad profesional.

\section{DE LA ÉTICA A LA ÉTICA PROFESIONAL}

¿Cómo va la ética en nuestro país? ¿Es sensato seguir hablando de ética en nuestro país cuando encontramos un déficit moral en todos los niveles? Por eso algunos pueden

- Magister en Filesofia con mención en Historia de la Filosofia. Profesor Asociado de la Facultad de Letras y Ciencias Humanas de la UNMSM. Investigador del Instituto de Investigaciones Humanisticas de la FLCH/UNMSM. Miembro de la Sociedad Peruana de Filosofía. 
sostener que hablar de ética en nuestro país es como hablar de la "dentadura del pollo". Sin embargo, a pesar de que los hechos sean así, no debemos dejarnos aplastar por ellos.

¿Es racional seguir hablando de ética en este contexto? Claro que sí, porque el hombre, como diría Kant, es un ser metafísico por naturaleza, es decir, que se resiste a someterse a los hechos fríos y opacos. ¿Por qué es posible seguir hablando de ética? Porque los seres humanos, a pesar de nuestros condicionamientos, hacemos opciones fundamentales. Las dos principales son una opción personal y una opción social. Con la primera podemos tomar la decisión de cultivar nuestro ser, es decir, comprometernos en lo que Mill denominaba el desarrollo de las capacidades intelectuales y morales. La ética tiene que ver con el bien personal, la autorrealización o felicidad. Con la segunda, tomamos la decisión de realizar el bien común, para lo cual participamos en una actividad comunitaria. De lo contrario, nuestra existencia social se pone en peligro, porque el bien común abarca la realización de las necesidades indispensables para vivir humana y dignamente. Ambas opciones son en realidad una misma opción que se expresa de dos maneras. Es decir, que la ética tiene que ver con el compromiso de ser una "buena persona», la cual pasa por la realización del bien común y la búsqueda de la plenitud de nuestra existencia.

Sin embargo, contra la concepción liberal tradicional, dicha opción requiere de condiciones previas que la hacen posible, es decir, no es un acto espontáneo ni esporádico. Entre las condiciones previas están el marco de referencia cultural, el saber práctico y la sensibilidad. Curiosamente, la búsqueda de esas condiciones es ya una búsqueda ética. Esa búsqueda nos lleva a interrogar, a cuestionar nuestro marco de referencia, especialmente la referida a la corrupción. ¿Cuáles son sus causas? ¿En qué nos involucra? ¿Cómo afrontarla o enfrentarla? Requerimos, pues, cuestionar, interrogar, y al hacerlo estamos planteando una opción ética. Interrogantes que nos llevan a la búsqueda de la vida buena. La interrogación y la búsqueda son entonces dos componentes indispensables de la vida ética. De lo contrario nos queda la imposición y el sometimiento al poder económico y político, que son dos formas que distorsionan las actividades sociales, entre ellas las profesionales. Éste es el marco mínimo para hablar de las éticas profesionales. Ahora, veamos un poco sobre la naturaleza de esta actividad pública denominada profesión.

\section{EL DEVENIR DE LAS PROFESIONES}

La cultura y la sociedad premoderna habían estructurado las profesiones a partir de una cosmovisión, donde jerárquicamente el orden divino ocupaba el primer lugar y como representación suya se encontraba el orden político, finalmente el orden personal. Para garantizar dicho orden estaban el sacerdote que cuidaba del alma divina y de los asuntos divinos, el jurista para cuidar de la sociedad mediante leyes, el médico para el cuidado del cuerpo. Por ello, el sacerdote, el médico y el jurista asumen sus actividades a partir de juramentos en los cuales prometen obedecer determinadas reglas. Por esa profesión o declaración fueron conocidas como las primeras profesiones.

El término "profesional" y su respectiva valoración son algo que surge en la época moderna, debido al proceso de secularización donde la vida mundana y sus actividades forman un campo distinto de la fe. El calvinismo, como momento intermedio y como lo ha sostenido Max Weber, había visto en las actividades profesionales el camino de salvación o condenación de una persona. Si había tenido éxito profesional indicaba que estaba salvada; si había fracasado, entonces estaba condenado. Ello permitió que la modernidad sea un proceso de «afirmación de la vida corriente" (Taylor), abriéndose un espacio a la revalorización de nuevas actividades humanas. Así, la cultura moderna asumió ciertas actividades como "profesiones", actividades seculares, mundanas, sin relación con la religión. De esa manera, nuevas actividades pasaron a ser profesiones, como ingeniero (de distintas especies), economistas, contadores, etc. Además, se incluyó en esta 
denominación medios de vida anteriores como médico, abogado, profesor, etc. Ahora, paralelo a la profesión de fe (profesar una fe), estaba el profesar una actividad, es decir, el consagrarse o comprometerse con una actividad y sus principios. Por eso se dice que la profesión aparece como una proclamación pública; no es una actividad secreta sino abierta y tiene el respaldo de la sociedad.

\section{3. ¿QUÉ ES UNA PROFESIÓN?}

Con la pregunta de lo que es la profesión no queremos hacer una simple descripción, sino que estamos preguntando por el sentido que tiene ésta. Max Weber, en su obra clásica La ética protestante y el espíritu del capitalismo, definía la profesión del siguiente modo:

la actividad especializada y permanente de un hombre que, normalmente, constituye para él una fuente de ingresos $y$, por tanto, un fundamento económico seguro de su existencia. (Weber 1985, 82)

Esta definición acentúa dos características de las profesiones: su carácter individual y económico. Las profesiones son un medio de subsistencia de los individuos. Eso hace de las profesiones un "instrumento individual" de ganar dinero. Sin embargo, como lo han señalado otros autores, este enfoque resulta muy limitado, porque olvida los fines y la actividad social de las profesiones. Adela Cortina, filósofa española, hace tres observaciones a esta definición:

a) La finalidad de la profesión no se limita a la "fuente de ingresos», es decir, a una finalidad subjetiva, sino que la profesión misma tiene su finalidad. El logro de dicha finalidad es lo que le da sentido y legitimidad social a la profesión, por ello la misma sociedad puede reclamarle que lo realice así como reclamar su calidad.

b) La profesión, además de ser una actividad individual, es una actividad colectiva, que expresa una cierta comunidad profesional que tienen los mismos fines, utilizan un lenguaje común, con métodos semejantes y con un ethos propio. Es decir, así como hay un ethos (carácter, modo de ser) personal, también existe un ethos de la profesión.

c) Por lo anterior, «el ingreso en una actividad $y$ en una comunidad profesional determinadas dota al profesional de una peculiar identidad y genera en él un peculiar sentido de pertenencia" (Cortina 2000, 15). Esto tiene que ver con que la profesión es una forma de afirmación de la sociedad civil frente al poder político, así como una afirmación de un espacio público frente a otros como el económico o religioso.

Por lo anterior, basada en las ideas de Macintyre, una definición más integradora de lo que es una profesión es la que ofrece Cortina. Una profesión es:

... una actividad social cooperativa, cuya meta interna consiste en proporcionar a la sociedad un bien específico $e$ indispensable para su supervivencia como sociedad humana, para lo cual se precisa el concurso de la comunidad de profesionales que como, tales se identifican ante la sociedad. (Cortina 2000, 15)

A partir de esta definición podemos darnos cuenta del sentido de una profesión. En primer lugar, una profesión busca realizar un bien o finalidad, que es indispensable para la vida social. Pero su realización exige el cultivo de hábitos o excelencias por parte del sujeto y de la acción que realiza. Finalmente, la actividad profesional no es una actividad aislada sino comunitaria, la cual le da una identidad profesional. Veremos más adelante estos componentes.

A pesar de que la palabra "profesión" tiene múltiples usos - porque sirve para designar tanto a un médico, un profesor, como para un vendedor, un futbolista y hasta suele hablarse de un delincuente profesional-, la definición propuesta puede ayudarnos a discernir cuando estamos en presencia de una profesión. Lo que sucede es que en nuestra época se han difuminado los antiguos límites entre 
"profesiones» y "oficios». Para darnos cuenta de esta complejidad de profesiones, veamos una posible clasificación. Antonio Peinador ha clasificado las profesiones teniendo en cuenta dos criterios: por razón de la actividad y por razón de los fines de cada profesión (Peinador 1962, 8-10):

\section{Por la actividad}

Manuales: predomina el trabajo corporal, manual o mecánico. También denominadas oficios.

Liberales: predomina el trabajo de la inteligencia. Denominada profesión propiamente tal.

\section{Por los fines}

Derecho a la vida fisica:

a) en un plano inferior: cocinero, zapatero, sastre, carpintero, labrador, artesano, etc.;

b) en un plano superior: técnicos e investigadores de laboratorio, industriales, comerciantes, banqueros, etc.

Derecho al cultivo progresivo e ilimitado de Ia inteligencia: científico, teólogo, filósofo, artista, pedagogo, etc.

Derecho a vivir como individuos y miembros de la sociedad dentro del orden jurídico y moral: político, sociólogo, abogado, juez, notario, historiador, novelista, religioso, etc.

En cualquier caso, para que algo sea considerado profesión o actividad profesional, dicha actividad debe estar comprometida al logro del bien común, para lo cual requiere el cultivo de ciertos hábitos o virtudes, en un contexto comunitario. De ese modo, el componente ético no es un añadido o un agregado que no afecta la esencia de la profesión. Aclaremos más estos componentes.

\section{LA PROFESIÓN COMO ACTIVIDAD CON FINALIDAD}

La actividad profesional es una actividad social que contiene fines o bienes internos. Son los fines los que dan orientación y sentido a las prácticas profesionales. La importancia de los fines la podemos encontrar en la definición de "profesión" que nos da Antonio Peinador. La profesión es:

la aplicación ordenada y racional de parte de la actividad del hombre al conseguimiento de cualquiera de los fines inmediatos y fundamentales de la vida humana. (Peinador 1962, 2).

Entonces, la profesión no es una simple actividad u ocupación, sino que debe estar orientada hacia un fin noble, es decir, el servicio a los demás: el curar, el enseñar, el informar, la convivencia justa, etc.; las profesiones no tienen sentido en sí mismas sino por los bienes internos que contienen. Por eso, no se puede entender lo que es una profesión si no se entiende que ésta tiene en su interior una función social: el bienestar común. De esa manera, el profesional es aquel hombre

...puesto al servicio de los demás, dentro del engranaje social, actuando con carácter público y comprometiéndose, en cuanto responsable de sus actos, ante Dios y ante su conciencia; $y$, además, ante aquellos a quienes sirve y de quienes se beneficia por ley de reciprocidad. (Peinador 1962, 7)

Así, una ética profesional requiere reconocer el bien interno que busca realizar determinada profesión; de lo contrario la actividad no tendrá sentido y legitimidad social. Esta forma de entender las acciones se enmarca en la tradición aristotélica. Para el estagirita, toda actividad tiene una finalidad, la cual constituye su bien. Sin embargo, no todos los fines son iguales. Hay fines que son internos a las actividades, es decir, en la realización de la actividad se encuentra la realización de la finalidad. Es a esto a lo que Aristóteles denomina praxis, mientras que hay fines que son externos a las actividades, por lo cual éstas se convierten en medios para lograr los fines. Es a esto a lo que Aristóteles denomina poiesis. En época reciente, Maclntyre 
ha tomado dichos términos para definir la práctica. Sostiene Maclntyre:

Por "práctica» entenderemos cualquier forma coherente y compleja de actividad humana cooperativa, establecida socialmente, mediante la cual se realizan los bienes inherentes a la misma mientras se intenta lograr los modelos de excelencia que le son apropiados a esa forma de actividad y la definen parcialmente, con el resultado de que la capacidad humana de lograr la excelencia y los conceptos humanos de los fines y bienes que conlleva se extienden sistemáticamente. (Macintyre 1987, 233)

Los elementos que componen una práctica son: i) el que sea una actividad cooperativa, ii) establecida socialmente, iii) busca la realización de los bienes internos, iv) el logro de esos bienes internos produce excelencias o virtudes en los sujetos que las realizan. No está sosteniendo el autor una visión medieval de estatus y roles predeterminados, sino que en tanto actores sociales ya nos encontramos realizando alguna actividad cooperativa. Somos funcionarios públicos, profesores, empleados de una empresa, profesionales en ejercicio, choferes, padres de familia, etc. Cada actividad tiene bienes internos cuya realización hace que ella tenga sentido. Si soy médico y me aprovecho de mi condición ante la debilidad de un enfermo, entonces no realizo el bien interno de la medicina, que es curar.

Maclntyre reconoce que existen también bienes externos legítimos que se obtienen de las prácticas, como el dinero, el reconocimiento, etc., pero ellos no son el sentido de la actividad. Justamente la corrupción de una actividad se produce cuando se reemplazan los bienes internos por los externos. Entonces tenemos que el profesor ya no enseña bien porque le pagan poco, el comisionado no cumple los reglamentos porque aprovecha su cargo para realizar sus intereses personales, el policía ya no protege a la ciudadanía y prefiere la coima, el abogado ya no sirve a la justicia sino a quien paga más, al político ya no le interesa el bien común sino los negocios de grupos e intereses partidarios, etc. Los bienes externos son legítimos, pero en tanto sean subordinados a los bienes internos; de lo contrario la actividad profesional deja de tener sentido y legitimidad social.

Sin embargo, las exigencias sociales y los cambios científicos y tecnológicos muchas veces nos obligan a redefinir los bienes internos de nuestras actividades profesionales. Por ejemplo, los avances de la medicina y las necesidades sociales hacen que la medicina ya no tenga sólo como finalidad el curar, sino que ahora agrega la promoción de la salud y la prevención de las enfermedades. Y es que el concepto mismo de salud ha sido redefinido, más de acuerdo con una visión humana integral. Por otro lado, la globalización y la internet hacen que los profesionales bibliotecarios también requieran redefinir sus finalidades. Cuando esto sucede, se abre un necesario periodo de debate y reflexión, en los cuales también deberían participar los afectados de las actividades profesionales.

Una observación adicional. ¿Cuál es el lugar del bien personal o autorrealización en la actividad profesional? La realización de los bienes internos forma parte de nuestra realización personal, de su proyecto de felicidad. Y es que el "horizonte de plenitud» (Etxeberria) hace referencia a la vida humana como totalidad. Sin embargo, esto plantea serios problemas. La sociedad moderna tiende a fragmentar la vida humana, a no asumirla integralmente. Por ello, la vivencia de las actividades profesionales suele ser asfixiante, donde los sujetos estresados pueden ser eficientes, pero no se sienten felices. El problema no es de las actividades profesionales mismas, sino de la finalidad del sistema económico: la ganancia. Por realizar la finalidad del capitalismo, los individuos en sus actividades profesionales suelen ser explotados al punto de no encontrar sentido a su profesión ni a su vida. Es ahí donde el poder político y las organizaciones profesionales deberían reducir al máximo las injusticias que produce el sistema económico. 


\section{LA EXCELENCIA COMO MODO DE REALIZAR EL FIN}

¿Qué es lo que pueden realizar los bienes internos a las prácticas profesionales? Siguiendo los lineamientos aristotélicos, podemos sostener que son las virtudes o excelencias (aretai). Las virtudes profesionales son la parte central de las éticas profesionales, porque ellas son las formas como se realizan los fines de las actividades profesionales.

Sin embargo, otros autores ponen el acento en las normas, es decir, en el "momento deontológico" (Etxeberria). Una definición en ese sentido de ética profesional la podemos encontrar en González, quien sostiene que la ética profesional es

la reflexión sistemática sobre las normas morales que regulan el comportamiento en la actividad profesional (González 1996, 277)

Pero la "reflexión sistemática» sobre las normas puede convertir la ética profesional en una deontología, limitando su valor. Para que no ocurra ello, es necesario que entendamos las normas morales del profesional no sólo como mínimos legales que está obligado a cumplir, sino como formas de promover las virtudes o excelencias de las acciones. No se trata entonces sólo de revisar y renovar las normas morales de los códigos de ética, sino de promover las virtudes necesarias para lograr los fines específicos de la profesión.

Por lo anterior, dejando de lado por un momento los códigos de ética, es necesario señalar que "ética profesional» no significa tanto la imposición o aceptación de normas, sino que toda práctica profesional conlleva virtudes o "excelencias" sin las cuales tales prácticas se corrompen. Por ello, Maclntyre ha acertado al entender las virtudes como cualidades adquiridas necesarias para realizar los bienes internos de las prácticas; su carencia impide lograr tales bienes. (Maclntyre 1987, 237).

La virtud no es un asunto privado, íntimo, cualidad que algunas personas conservadoras o bienintencionadas tienen. Es un asunto personal e interpersonal, subjetivo e intersubjetivo, individual y colectivo. $Y$ ese nexo se encuentra en las acciones, en las prácticas en las cuales nos encontramos con otros para realizar los bienes internos. Existe, pues, en la definición de virtud de Maclntyre una indesligable relación entre virtudes y bienes internos. Sin virtudes no es posible la realización de los bienes internos de las actividades, así como sin realización de los bienes internos no es posible virtud alguna.

Sin duda, esto sugiere que no todas las actividades requerirán las mismas virtudes. El policía, el obrero, el empleado y el profesor requieren virtudes distintas para lograr sus bienes internos. Pero Macintyre se percata de que esto produciría un politeísmo de virtudes inconexas, por lo que sostiene que aunque las prácticas sean distintas, podemos sostener un mínimo de virtudes (como la integridad) que surgen de la búsqueda de la vida buena, de asumir nuestra existencia como un todo unitario. El mismo problema es visto por Camps, cuando sostiene que en la sociedad de profesionales, cada profesión tiene virtudes específicas distintas de las virtudes públicas (como solidaridad, responsabilidad, tolerancia). Y señala algo que suscribo plenamente:

La profesionalidad será una virtud pública en la medida en que sirva a los intereses comunes de la sociedad. No en la medida en que sirva sólo al mantenimiento $y$ conservación de los roles, funciones $y$ corporaciones existentes. $Y$ será una virtud privada en la medida en que ayude al individuo a serlo realmente, a ser autónomo y no esclavo de sus actividades. (Camps 1993, 105)

Volviendo a la pregunta por las «virtudes de una profesión", ésta significa lo mismo que la pregunta de cuándo alguien es un «buen profesional». No son dos cosas distintas. Alguien es buen profesional cuando realiza con destreza y responsabilidad la finalidad de su actividad. Por ejemplo, alguien es un buen profesor cuando realiza el bien interno, el cual 
es educar. No es un buen profesor aquel que maltrata a los alumnos física o psicológicamente, si impone su criterio de autoridad, si no sabe llegar a los alumnos, etc. Según González, las virtudes de un buen profesional son la productividad (que encierra la capacidad de producir, la actitud de producir y el nivel de eficiencia), la creatividad y la superación. Sin embargo, no siempre todas las profesiones requieren de las mismas virtudes. Para resolver la pregunta sobre cuáles son las buenas prácticas o virtudes que debe cultivar el profesional, deben participar tanto los mismos profesionales como los afectados o beneficiarios o usuarios. Sobre esto, Cortina sostiene:

Los usuarios son los que experimentan la calidad del servicio prestado y, aunque no conocen la trama interna de la profesión. resultan indispensables para determinar qué prácticas producen un servicio de calidad y cuáles no. De ahí que hoy en día los colegios profesionales no puedan ser cerrados, no puedan diseñar sus códigos ni componer comités sin contar con los ciudadanos corrientes, con los beneficiarios actuales o virtuales del servicio que prestan a la sociedad. En este punto deben transformarse radicalmente. (Cortina 2000, 24)

A veces perdemos de vista el fin que perseguimos, y cuando lo tenemos en cuenta tendemos a diferenciarlo de las virtudes y creemos que éstas son simples medios. Pero las virtudes no son simples medios para lograr algo. La práctica de las virtudes implica ya la realización de la "finalidad" de la profesión. El fin de una profesión no es algo subjetivo y alejado en un futuro incierto, sino que está contenido en la práctica correcta de la profesión. Cuando los fines y los medios los ponen la sociedad y su economía, no permite la correcta práctica de una profesión. Por ejemplo, al médico se le exige productividad y eficiencia, pero se ve obligado a reducir el tiempo de diálogo con sus pacientes descuidando tanto un examen cuidadoso como el trato humano que requieren los enfermos. Por lo anterior, estamos de acuerdo con González cuando dice que las virtudes que encarna el buen profesional son distorsionadas por la fetichización de la economía, la deshumanización de las relaciones sociales y el olvido de que la vida es el valor supremo (González 1996, 289-290). De ese modo, la sociedad en que vivimos convierte en fines los medios y los medios en fines, no permitiendo un buen ejercicio de la profesión.

\section{LA PROFESIÓN COMO ACTIVIDAD COMUINITARIA}

Las profesiones son expresiones de la "actividad humana cooperativa», es decir, las prácticas profesionales son comunitarias. Esto por lo menos en tres sentidos.

En primer lugar, porque el profesional comparte con otros profesionales un lenguaje común, un método y hasta un modo de ser. La transmisión y la renovación del saber especializado se realizan dentro de comunidades profesionales. Por eso resulta extraño, por decir lo menos, que un profesional sociólogo, por ejemplo, dicte cursos de especialidad para administradores. Ello sólo se admite cuando el sociólogo también tiene formación de administrador.

En segundo lugar, la comunidad profesional se convierte en el referente del individuo profesional, porque le da identidad y pertenencia. Este aspecto no es poca cosa, sino que tiene que ver con la identidad de una persona, pues así como tenemos identidad religiosa, nacional, política, también tenemos identidad profesional.

En tercer lugar, porque la comunidad de profesionales hace suya la tarea de realizar la finalidad de dicha práctica. En otras palabras, las profesiones sirven a la sociedad para realizar bienes específicos, pero to hacen de forma institucionalizada. Por ello, los profesionales forman corporaciones, colegios profesionales. Esto tiene ventajas y desventajas. Los colegios profesionales sirven para autorizar y fiscalizar la práctica profesional, procurando que llegue a dar un servicio de calidad. Además, vigilan que dicha 
actividad sea ejercida por personas que hayan pasado por un previo periodo de formación, especialmente si son actividades con gran responsabilidad social. Por ello es que los colegios profesionales son la instancia que autoriza el ejercicio profesional.

Sin embargo, este sentido de solidaridad puede degenerar en "corporativismo", que se manifiesta en el encubrimiento de las actividades ilícitas cometidas por los profesionales. Se busca salvar el cuerpo, el colegio, protegiendo a individuos profesionales incompetentes o inmorales o negligentes, dejando de tener en cuenta los "mínimos niveles de calidad profesional" (Vielva). También se manifiesta en la exigencia o defensa de privilegios corporativos, relegando la "solidaridad universal» por la "solidaridad grupal». Como lo señala Cortina, la «elevada misión» que justifica privilegios a determinadas profesiones sólo tiene sentido dentro de una visión gremial de la sociedad, muy típica de la edad media. Pero en una sociedad con estado de derecho que por principio defiende la igualdad de la ley para todos los grupos sociales, tal actitud resulta anacrónica. En cualquier caso, el corporativismo pierde el sentido de la comunidad de profesionales.

Finalmente, el ejercicio de las profesiones puede hacer que se amolden a las instituciones públicas o privadas sólo respetando las normas y procedimientos de la institución y caen en la rutina a tal punto que dejan de tener en cuenta la misión que tienen. Es lo que se ha llamado "burocratismo». En palabras de Cortina:

Frente al ethos burocrático de quien se atiene al mínimo legal, pide el ethos profesional la excelencia, porque su compromiso fundamental no es el que les liga a la burocracia, sino a las personas concretas, a las personas de carne y hueso, cuyo beneficio da sentido a cualquier actividad e institución social. (Cortina 2000, 28)

\section{LA PROFESIÓN COMO EXPRESJÓN DE LA SOCIEDAD CIVIL}

Es célebre la distinción hegeliana de que la eticidad está conformada por la familia, la sociedad civil y el Estado. Sin embargo, entendió la sociedad civil como expresión de las actividades económicas, es decir, del mercado. Hoy no es posible sostener que la sociedad civil, esté compuesta básicamente por el mercado y que sea lo único que hace frente al espacio político.

La sociedad civil también es el ámbito de las actividades profesionales, la opinión pública, las asociaciones cívicas y, sin duda, las organizaciones económicas. Lo cual quiere decir que las profesiones juegan un papel importante en la sociedad civil, por ello su necesidad de afirmarse como espacio público diferente de las actividades económicas y de las políticas, que hoy pretenden ocupar todo el espacio publico. Tan importante es el espacio profesional en la sociedad civil que sin ella ninguna actividad política y empresarial podría realizar sus actividades con óptimos resultados.

Con conciencia de ello, los Colegios Profesionales pueden jugar un rol más activo dentro de la sociedad peruana, superando de ese modo el corporativismo que les hace perder su sentido. Las comunidades profesionales tienen una responsabilidad social porque es la misma sociedad la que hace necesaria su existencia. Por pertenecer a la sociedad y porque ella hace necesaria las actividades profesionales, ella también está autorizada o legitimada para exigir a los profesionales que cumplan con la realización de los fines o bienes específicos.

\section{A MODO DE CONCLUSIÓN}

Podemos resumir y concluir lo trabajado a partir de tres aspectos que inevitablemente tocan la vida profesional y tienen contenido ético, como son los aspectos social, económico y personal.

a) El aspecto social. Las profesiones tienen por función satisfacer determinadas necesidades de las personas, de los miembros de una sociedad. Esta dimensión de servicio que todo profesional presta es marcadamente una dimensión moral y es el centro de su actividad. No existe un 
profesional que no preste un servicio a la sociedad justamente ésa es su razón de ser. $Y$ ese servicio lo hace de manera comunitaria, generalmente agrupados en colegios profesionales. Para un mejor logro de los bienes internos de las actividades profesionales, estos colegios exigen actualización, mayor profesionalización y exigen (o deberían exigir) el respeto de los códigos de ética de la profesión. Aunque siempre existe la duda de si los colegios profesionales realmente funcionan como una comunidad o como una corporación.

Este aspecto social también se refiere a la influencia que tienen las profesiones en la sociedad. Una profesión no es un ente autónomo culturalmente, sino que recibe influencias y a su vez influye culturalmente en la sociedad. Por eso, la comunidad de profesionales también es responsable del estado actual de la sociedad, sea porque no cumple con su rol ni genera modelos de excelencia. Ha sido el caso del periodismo en la década turbia del fujimorismo, donde los periodistas inventaban la noticia, se vendían al poder político, difamaban la honra de las personas, degenerando la práctica del periodismo. Creo que no se puede dudar de que eso haya impactado negativamente en la mentalidad nacional.

b) El aspecto económico. Toda profesión tiene una dimensión económica tanto a nivel social como personal. La profesión presenta en primer término un carácter marcadamente económico, puesto que la economía también se refiere a las necesidades humanas y al modo de satisfacerlas. En efecto, las profesiones se desarrollan en el campo de la economía, obedecen a causas económicas y producen efectos de la misma clase. (Álvarez 1957, 171)

Así, los colegios profesionales también tienen que ver con esta función económica, porque los colegios sirven para que sus miembros puedan acceder al mercado de trabajo de forma organizada y vigilada. Pero no se puede negar que aparte de esa 'iniciación' en los beneficios del mercado que el colegio (intermediario entre el gobierno y la clientela) ofrece, puede funcionar $y$ de hecho funciona un mecanismo de control moral interno, a nivel de los colegiados. (Regal 1988, 195)

Sin embargo, el sistema económico ha convertido la sociedad en una "sociedad de productores", desvalorizando o cuestionando como actividad profesional a aquellas que no producen lo que el sistema requiere. Es el caso de profesiones como la Filosofia, la Literatura, la Teología, el Arte, etc. No obstante, mientras no se entienda el producto en sentido reducido (técnico-científico), existe espacio suficiente para justificar la existencia de todas estas actividades. Los bienes que una sociedad requiere no sólo son económicos, sino existe toda una gama que está en relación con las necesidades espirituales, intelectuales, estéticas y vitales.

Además, este aspecto económico de la profesión no sólo significa que los profesionales accedan al mercado de trabajo, sino que la actividad profesional se ha convertido en el medio económico de subsistencia de los profesionales. La profesión nos proporciona los medios económicos de satisfacer las necesidades privadas, las personales y las domésticas. La actividad profesional es hoy la fuente normal de ingresos de todo individuo que no vive a costa de los demás. (Álvarez 1957, 173)

Sin duda, este aspecto también se relaciona con el siguiente, pero debido a las condiciones económicas del sistema capitalista, las personas no suelen ver al trabajo, y a la actividad profesional en particular, como una dimensión que dé sentido a sus vidas. Es visto como medio para conseguir riqueza y poder, no como parte de su realización personal. La alienación de la economía, del trabajo y de la actividad profesional son factores que deben ser tenidos en cuenta para pensar una ética profesional.

c) El aspecto personal. Por último, las profesiones también incluyen un aspecto 
personal. Esto en un doble sentido. Por un lado, la práctica profesional requiere de cierta vocación o aptitudes para prestar un mejor servicio. Por otro lado, las profesiones moldean generalmente todas las demás áreas de la vida de un profesional.

La vida de cada uno se organiza alrededor de su profesión de tal suerte que si ésta falta suele aquélla desarticularse. Semejante organización afecta a la vez la vida privada y pública del individuo. Hábitos, juicios, modos de pensar y hacer, formas de acción internas y externas dependen de la profesión y por ella se explican. Tipos de vida familiar, estilos de amistad también se derivan de la profesión en gran medida. (Álvarez 1957, 172-173)

Sostiene Camps que la vida profesional puede ser fuente de disfrute y reconocimiento. El problema es con la "profesionalización absoluta», es decir, está cuando la actividad profesional ocupa toda la vida personal, fragmentando su vida y viviendo en un espacio reducido. El trabajo se convierte en su único mundo, generando un individualismo acorde con las necesidades del sistema económico pero no con las mejores realizaciones humanas. El otro aspecto negativo de la actividad profesional es la "pérdida de autonomía», es decir, cuando lo único que motiva a la actividad son los bienes externos como el dinero, el éxito, el poder, olvidando los bienes internos (Camps 1993, 102-104).

En síntesis, como se habrá entendido, la moral está presente en cada aspecto de una profesión: en lo social, económico y personal, dando sentido y legitimidad a la actividad profesional. Más aún, la moral es la que enlaza cada dimensión, porque la moral es la forma de vida que tenemos. Así, cada aspecto no está aislado del otro. Cuando uno de los tres aspectos resulta enajenado, perjudicado por la sobrevaloración de uno de ellos, vienen los problemas de inmoralidad, pérdida del sentido de la actividad, frustración, formación de grupos de poder, etc.

La ética profesional tiene, pues, la tarea de articular los distintos aspectos, tarea nada fácil pero que compromete totalmente. ¿Tendremos los profesionales el valor de asumir ese reto? Sea que se asuma o no se asuma, ya se ha optado y en ambos casos habrá consecuencias de las que deberemos responsabilizarnos.

\section{BIBLIOGRAFÍA}

- ÁlVAREZ, Joaquín. Ética de nuestro tiempo. México: UNAM. 1957.

- CAMPS, Victoria. Virtudes públicas. Madrid: Espasa Calpe. 1993.

- CORTina, A. Hasta un pueblo de demonios. Ética pública y sociedad. Madrid. Taurus. 1998.

- CORTINA, A. Y CONILl,J. (Dirs.). 10 palabras clave en Ética de las profesiones. Navarra: Verbo Divino. 2000.

- GONZÁLEZ, Luis José. Ética. Santafé de Bogotá: El Búho. 1996.

- MACINTYRE, Alasdair. Tras la virtud. Madrid: Cátedra. 1987.

- PEINADOR, A. Tratado de moral profesional. Madrid: BAC. 1962.

- REGAL, Bernardo. Fundamentos de ética profesional. Lima: Universidad de Lima. 1988.

- VIELVA, Julio. Ética profesional de la enfermería. Bilbao: Descleé De Brouwer. 2002.

- WEBER, Max. La ética protestante y el espíritu del capitalismo. Buenos Aires: Orbis. 1985. 